PROCEEDINGS OF THE

AMERICAN MATHEMATICAL SOCIETY

Volume 137, Number 7, July 2009, Pages 2425-2435

S 0002-9939(09)09804-9

Article electronically published on February 12, 2009

\title{
ON CLOSED SETS WITH CONVEX PROJECTIONS UNDER SOMEWHERE DENSE SETS OF DIRECTIONS
}

\author{
STOYU BAROV AND JAN J. DIJKSTRA
}

(Communicated by Nigel J. Kalton)

\begin{abstract}
Let $k, n \in \mathbb{N}$ with $k<n$ and let $\mathcal{G}_{k}\left(\mathbb{R}^{n}\right)$ denote the Grassmann manifold consisting of all $k$-dimensional linear subspaces in $\mathbb{R}^{n}$. In an earlier paper the authors showed that if the projections of a nonconvex closed set $C \subset \mathbb{R}^{n}$ are convex and proper for projection directions from some nonempty open set $\mathcal{P} \subset \mathcal{G}_{k}\left(\mathbb{R}^{n}\right)$, then $C$ contains a closed copy of an $(n-k-1)$-manifold. In this paper we improve on that result by showing that that result remains valid under the weaker assumption that $\mathcal{P}$ is somewhere dense in $\mathcal{G}_{k}\left(\mathbb{R}^{n}\right)$.
\end{abstract}

\section{INTRODUCTION}

Let $k, n \in \mathbb{N}$ with $k<n$ and let $\mathcal{G}_{k}\left(\mathbb{R}^{n}\right)$ denote the Grassmann manifold consisting of all $k$-dimensional linear subspaces of $\mathbb{R}^{n}$. If $A$ is a subset of a topological space, then $\bar{A}$ is the closure of $A$ and int $A$ is the interior of $A$. If $B, C \subset \mathbb{R}^{n}$ and $\mathcal{P} \subset \mathcal{G}_{k}\left(\mathbb{R}^{n}\right)$, then $B$ and $C$ are called $\mathcal{P}$-imitations of each other if $B+P=C+P$ for each $P \in \mathcal{P}$. If $\overline{B+P}=\overline{C+P}$ for each $P \in \mathcal{P}$, then $B$ and $C$ are called weak $\mathcal{P}$-imitations of each other. Also, a $k$-plane in $\mathbb{R}^{n}$ is an affine subspace of $\mathbb{R}^{n}$ of dimension $k$.

Now, let $B$ be a closed convex subset of $\mathbb{R}^{n}$ that contains no $k$-plane and let $\mathcal{P} \subset \mathcal{G}_{n-k}\left(\mathbb{R}^{n}\right)$ be open such that $B$ is not a $\mathcal{P}$-imitation of $\mathbb{R}^{n}$. In this setting the authors showed in [3. Theorem 18] that if $C$ is a closed weak $\mathcal{P}$-imitation of $B$ with $C \neq B$, then $C \cap B$ contains a closed copy of a $(k-1)$-manifold. The main purpose of this paper is to show that the above result remains valid if we weaken the assumption on $\mathcal{P}$ from open to somewhere dense in $\mathcal{G}_{n-k}\left(\mathbb{R}^{n}\right)$. Our main result reads as follows.

Theorem 1. Let $k, n \in \mathbb{N}$ with $k<n$, let $B$ be a closed convex subset of $\mathbb{R}^{n}$ that contains no $k$-plane, and let $\mathcal{P}$ be a subset of $\mathcal{G}_{n-k}\left(\mathbb{R}^{n}\right)$ such that $B$ is not an (int $\overline{\mathcal{P}}$ )-imitation of $\mathbb{R}^{n}$. If $C$ is a closed weak $\mathcal{P}$-imitation of $B$ with $C \neq B$, then $C \cap B$ contains a closed set that is homeomorphic to either

(i) $\mathbb{R}^{k-1}$ or

(ii) $S^{i} \times \mathbb{R}^{k-i-1}$ for some $i \in\{1,2, \ldots, k-1\}$.

Received by the editors April 28, 2008, and, in revised form, October 11, 2008.

2000 Mathematics Subject Classification. Primary 52A20, 46A55, 57N15.

Key words and phrases. Convex projection, shadow, hyperplane, imitation, Grassmann manifold, proper mapping.

The first author is pleased to thank the Vrije Universiteit Amsterdam for its hospitality and support.

(C)2009 American Mathematical Society Reverts to public domain 28 years from publication 
Here $S^{i}$ stands for the $i$-dimensional sphere. Observe that [3, Theorem 18] corresponds to Theorem 1 with the additional assumption that $\mathcal{P}$ is open in $\mathcal{G}_{n-k}\left(\mathbb{R}^{n}\right)$. The method we use is to show that if $\mathcal{P}$ satisfies the premises of Theorem 1 , then int $\overline{\mathcal{P}}$ contains a nonempty open subset that satisfies the premises of 3 , Theorem 18]; see Theorem 13. Let us point out that our approach for proving the reduction of Theorem 1 1 to [3, Theorem 18] is sufficiently general so as to include the case that the ambient space is the separable Hilbert space $\ell^{2}$ so that the results are also of use for a forthcoming extension [4] of the results in [2] and [3] over $\ell^{2}$.

Theorem 1 deals with the retrieval of information about a geometric object from data about its projections, which places the result in the field of Geometric Tomography; see Gardner [10] for background information. As far as the history of the type of problems we consider is concerned, Borsuk [6] has shown that there exist Cantor sets in $\mathbb{R}^{n}$ such that all their shadows (projections onto hyperplanes) contain $(n-1)$-dimensional convex bodies. In contrast, Cobb [7] showed that every compaction $C$ in $\mathbb{R}^{n}$ with the property that all its shadows are convex bodies contains an arc. Dijkstra, Goodsell, and Wright 8 improved on this result by showing that such a $C$ must contain an $(n-2)$-sphere. Barov, Cobb, and Dijkstra 1 were subsequently able to construct an extension of that result over the class of unbounded closed sets and 2] concerns the Hilbert space variant of the problem. In [3] we showed that the results in [8] and [1] remain valid if we make the much weaker assumption that the collection of projection directions that produce convex shadows has a nonempty interior.

\section{DEFINITIONS AND PRELIMINARIES}

Throughout this paper $\mathbb{V}$ stands for a separable real Hilbert space with an inner product $x \cdot y$. Thus $\mathbb{V}$ is isomorphic to either an $\mathbb{R}^{n}$ or $\ell^{2}$. The norm on $\mathbb{V}$ is given by $\|u\|=\sqrt{u \cdot u}$ and the metric $d$ is given by $d(u, v)=\|v-u\|$. We denote by $\mathbf{0}$ the origin of $\mathbb{V}$ and $\mathbb{S}$ stands for the unit sphere in $\mathbb{V}$. Let $A$ be a subset of $\mathbb{V}$. We have that $[A]$ denotes the linear hull and $\langle A\rangle$ the convex hull of $A$. We define $A^{\perp}$ in the following way:

$$
A^{\perp}=\{v \in \mathbb{V}: v \cdot x=v \cdot y \text { for all } x, y \in A\} .
$$

Also, we define $\operatorname{codim} A=\operatorname{dim} A^{\perp} \in\{0,1, \ldots, \infty\}$. A plane in $\mathbb{V}$ is a closed affine subspace of $\mathbb{V}$; a $k$-subspace is a $k$-dimensional linear subspace of $\mathbb{V}$. The affine hull aff $A$ of $A$ is defined as the intersection of all planes that contain $A$. The geometric interior $A^{\circ}$ of $A$ is the interior of $A$ relative to the affine hull of $A$.

Definition 1. Let $\mathcal{K}(\mathbb{V})$ stand for all nonempty compact subsets of $\mathbb{V}$. Recall that the Hausdorff metric $d_{\mathrm{H}}$ on $\mathcal{K}(\mathbb{V})$ associated with $d$ is defined as follows:

$$
d_{\mathrm{H}}(A, B)=\sup \{d(x, A), d(y, B): x \in B \text { and } y \in A\} .
$$

We let $\mathcal{G}_{k}(\mathbb{V})$ stand for the collection of all $k$-subspaces of $\mathbb{V}$. Consider the ball $\mathbb{B}=\{v \in \mathbb{V}:\|v\| \leq 1\}$. We topologize $\mathcal{G}_{k}(\mathbb{V})$ by defining a metric $\rho$ on $\mathcal{G}_{k}(\mathbb{V})$ :

$$
\rho\left(L_{1}, L_{2}\right)=d_{\mathrm{H}}\left(L_{1} \cap \mathbb{B}, L_{2} \cap \mathbb{B}\right) .
$$

When $\mathbb{V}$ is finite-dimensional then $\mathcal{G}_{k}(\mathbb{V})$ is known as a Grassmann manifold.

The following lemma was proved for $\mathbb{R}^{n}$ in [3, Lemma 2]. The proof for $\ell^{2}$ is analogous. 
Lemma 2. Let $k \in \mathbb{N}$ with $k<\operatorname{dim} \mathbb{V}, \varepsilon>0, L \in \mathcal{G}_{k}(\mathbb{V})$, and let $v_{1}, \ldots, v_{k}$ be a basis for $L$. Then there is a $\delta>0$ such that for every set $F=\left\{v_{1}^{\prime}, \ldots, v_{k}^{\prime}\right\} \subset \mathbb{V}$ with $\left\|v_{i}^{\prime}-v_{i}\right\|<\delta$ for every $i$ we have $\rho([F], L)<\varepsilon$.

Now let $L$ be a plane in $\mathbb{V}$. A plane $H \subset L$ is called a $k$-hyperplane in $L$ if $\operatorname{dim}\left(H^{\perp} \cap L\right)=k$. In other words, a $k$-hyperplane is a plane with codimension $k$ in the ambient space. A hyperplane $H$ of $L$ is a plane of $L$ of codimension 1 . The two components of $L \backslash H$ are called the sides of the hyperplane $H$ and the union of $H$ with one of its sides is called a halfspace of $L$. We say that $H$ supports a subset $A$ of $L$ if $A$ is contained in a halfspace that is associated with $H$.

Definition 2. Let $B$ be a closed and convex set in $\mathbb{V}$. A nonempty subset $F$ of $B$ is called a face of $B$ if there is a hyperplane $H$ of aff $B$ that supports $B$ with the property $F=B \cap H$. Note that $F$ is also closed and convex and that $\operatorname{codim} F>\operatorname{codim} B$ whenever $\operatorname{codim} B$ is finite. If $F$ is a face of $B$ we write $F \prec B$. We say that a subset $F$ of $B$ is a derived face of $B$ if $F=B$ or there exists a sequence $F=F_{1} \prec F_{2} \prec \cdots \prec F_{m}=B$ for some $m$.

Definition 3. Let $\mathcal{P}$ be a collection of closed linear subspaces of $\mathbb{V}$. A hyperplane $H$ in $\mathbb{V}$ is said to be consistent with $\mathcal{P}$ if $H+P=H$ for some $P \in \mathcal{P}$. Let $B$ be a closed and convex subset of $\mathbb{V}$. A nonempty subset $F$ of $B$ is called a $\mathcal{P}$-face of $B$ if $F=B \cap H$ for some hyperplane $H$ of $\mathbb{V}$ that supports $B$ and that is consistent with $\mathcal{P}$. A derived $\mathcal{P}$-face is a derived face of a $\mathcal{P}$-face. If $k \in \mathbb{N}$ and $k<\operatorname{dim} \mathbb{V}$, then we define the set $\mathcal{E}^{k}(B, \mathcal{P})$ as the closure of

$$
\bigcup\{F: F \text { is a derived } \mathcal{P} \text {-face of } B \text { with } \operatorname{codim} F>k\} \text {. }
$$

Definition 4. Let $B, C \subset \mathbb{V}$ and let $\mathcal{P}$ be a set of closed linear subspaces of $\mathbb{V}$. $B$ and $C$ are called $\mathcal{P}$-imitations of each other if $B+P=C+P$ for each $P \in \mathcal{P}$. If $\overline{B+P}=\overline{C+P}$ for each $P \in \mathcal{P}$, then $B$ and $C$ are called weak $\mathcal{P}$-imitations of each other.

Definition 5. Let $L$ be a plane in $\mathbb{V}$. Then $\psi_{L}: \mathbb{V} \rightarrow L^{\perp}$ denotes the orthogonal projection along $L$ onto $L^{\perp}$ defined by the conditions $\psi_{L}(x)-x \in L^{\perp \perp}$ and $\psi_{L}(x) \in$ $L^{\perp}$ for each $x \in \mathbb{V}$. Note that if $\mathbf{0} \in L$, then $\left\{\psi_{L}(x)\right\}=L^{\perp} \cap(x+L)$.

Remark 1. Observe that $B$ and $C$ are (weak) $\mathcal{P}$-imitations of each other precisely if $\psi_{P}(B)=\psi_{P}(C)\left(\overline{\psi_{P}(B)}=\overline{\psi_{P}(C)}\right)$ for each $P \in \mathcal{P}$. If $B$ and $C$ are weak $\mathcal{P}$-imitations of each other, then $B$ and $C$ have precisely the same supporting hyperplanes that are consistent with $\mathcal{P}$, as follows. Let $H$ be a supporting hyperplane to $B$ such that $H+P=H$ for some $P \in \mathcal{P}$. Then $H$ supports $B+P$ and hence also $\overline{B+P}$ because halfspaces are closed. Since $\overline{B+P}=\overline{C+P}$ we have that $H$ supports $C$.

Lemma 3. Let $\mathcal{P}$ be a set of closed linear subspaces of $\mathbb{V}$ and let $B, C \subset \mathbb{V}$. If $C$ is a (weak) $\mathcal{P}$-imitation of $B$, then also $B \cup C$ is a (weak) $\mathcal{P}$-imitation of $B$ and if, in addition, $B$ is convex, then $\langle C\rangle(\overline{\langle C\rangle})$ is a (weak) $\mathcal{P}$-imitation of $B$.

Proof. Let $P \in \mathcal{P}$. If $B+P=C+P$, then $B+P \subset(B \cup C)+P=(B+P) \cup(C+P)=$ $B+P$ and for convex $B, C+P \subset\langle C\rangle+P=\langle C+P\rangle=\langle B+P\rangle=B+P=C+P$. If $\overline{B+P}=\overline{C+P}$, then $\overline{B+P} \subset \overline{(B \cup C)+P}=\overline{B+P} \cup \overline{C+P}=\overline{B+P}$ and for convex $B, \overline{C+P} \subset \overline{\overline{\langle C\rangle}+P} \subset \overline{\langle C+P\rangle} \subset \overline{\langle\overline{\langle+P}\rangle}=\overline{B+P}=\overline{C+P}$. 
A continuous map $f: X \rightarrow Y$ is called proper if the pre-image of every compactum in $Y$ is compact. Recall that in metric spaces a continuous map is proper if and only if it is closed and every fibre is compact; see Engelking 9, Theorem 3.7.18]. In particular, if $B \subset \mathbb{V}$ and a linear space $L \subset \mathbb{V}$ are such that the restriction $\psi_{L}\left\lceil B: B \rightarrow \mathbb{V}\right.$ is proper, then $\psi_{L}(B)$ and $B+L=\psi_{L}^{-1}\left(\psi_{L}(B)\right)$ are closed in $\mathbb{V}$. We shall use the following basic fact about proper maps; see [3, Lemma 6] or 9. Corollary 3.7.2 and Proposition 3.7.10].

Lemma 4. If $f: X \rightarrow Y$ and $g: Y \rightarrow Z$ are continuous, then $g \circ f: X \rightarrow Z$ is proper if and only if both $f$ and $g \uparrow f(X): f(X) \rightarrow Z$ are proper.

Remark 2. The following fact can be found in [11, §2.5] and [5, p. 93]. If $B$ is a closed convex set in $\mathbb{V}$, then there is a unique closed linear space $\mathcal{L}_{B} \subset \mathbb{V}$ such that $\operatorname{cs} B=B \cap\left(\mathcal{L}_{B}\right)^{\perp}$ is line-free and $B=\mathcal{L}_{B}+\operatorname{cs} B$. Observe that we have $\operatorname{cs} B=\psi_{\mathcal{L}_{B}}(B)$ and $B=B+\mathcal{L}_{B}$.

The following result is from [1, Lemma 4] and [2, Lemma 6].

Lemma 5. If $B$ is closed and convex in $\mathbb{V}$, then for every derived face $F$ of $B$ we have $F=F+\mathcal{L}_{B}$.

\section{PROPER PROJECTION MAPS}

This section consists of a series of lemmas that we need to prove our main results. Several of the lemmas are of independent interest as they deal with the properties of proper projections of convex sets.

Lemma 6. Let $B$ be a closed and convex set in $\mathbb{V}$ and let $P$ be a finite-dimensional linear subspace of $\mathbb{V}$. Then $\psi_{P}\lceil B$ is proper if and only if $(z+P) \cap B$ is bounded for some $z \in B$.

Proof. One direction is trivial because every fibre of a proper map is compact and $(z+P) \cap B$ is a fibre of $\psi_{P}\lceil B$. Assume that there is a $z \in B$ such that $(z+P) \cap B$ is bounded. We may assume that $z=\mathbf{0}$. Define

$$
\mathcal{A}=\left\{L: L \text { is a linear subspace of } P \text { such that } \psi_{L}\lceil B \text { is proper }\} .\right.
$$

Note that $\psi_{\{\mathbf{0}\}}$ is the identity function; thus $\{\mathbf{0}\} \in \mathcal{A}$. We may define $m=$ $\max \{\operatorname{dim} L: L \in \mathcal{A}\}$. It suffices to show that $m=\operatorname{dim} P$, so let us assume that $m<\operatorname{dim} P$. Choose an $m$-subspace $L$ of $P$ such that $\psi_{L}\lceil B$ is proper. Set $B_{L}=\psi_{L}(B)$ and note that $B_{L}$ is convex and closed in the space $V=L^{\perp}$. Let $u$ be a unit vector in $P$ that is perpendicular to $L$ and let $\ell$ be the line $\mathbb{R} u$. Put $L^{\prime}=L+\ell$, so $L^{\prime} \notin \mathcal{A}$. Since $\psi_{L^{\prime}}=\psi_{\ell} \circ \psi_{L}$ we have by Lemma 4 that it suffices to prove that $\psi_{\ell}\left\lceil B_{L}\right.$ is proper to arrive at the desired contradiction.

Since $\ell \cap B_{L} \subset \psi_{L}(P \cap B)$ and $P \cap B$ is bounded we have that $\ell \cap B_{L}$ is bounded as well. Thus we can find numbers $r_{1}, r_{2} \in \mathbb{R}$ such that $\ell \cap B_{L} \subset\left(r_{1}, r_{2}\right) u$ and $r_{1}<0<r_{2}$. Since $B_{L}$ is closed and convex and $r_{i} u \notin B_{L}$ we can find for $i=1,2$ the (unique) $B_{L}$-supporting hyperplane $H_{i}$ in $V$ so that $r_{i} u \in H_{i}$ and $d\left(H_{i}, B_{L}\right)=d\left(r_{i} u, B_{L}\right)>0$; see [12, p. 347]. Let $n_{i}$ be a normal vector to $H_{i}$ such that for every $x \in B_{L}$ we have $x \cdot n_{i}<r_{i} u \cdot n_{i}$. Substituting $x=\mathbf{0}$ we find $u \cdot n_{1}<0$ and $u \cdot n_{2}>0$.

To show that $\psi_{\ell}\left\lceil B_{L}\right.$ is proper let $C$ be a compact subset of $\left(L^{\prime}\right)^{\perp}$. Let $x \in C$ and let $y \in B_{L}$ be such that $\psi_{\ell}(y)=x$. Then $y=x+t u$ for some $t \in \mathbb{R}$ and 
$y \cdot n_{i}<r_{i} u \cdot n_{i}$ for $i=1,2$. Substituting $y$ in the inequalities and solving for $t$ we find

$$
r_{1}-\frac{x \cdot n_{1}}{u \cdot n_{1}}<t<r_{2}-\frac{x \cdot n_{2}}{u \cdot n_{2}} .
$$

Since $t$ is enclosed between two continuous functions that are defined on a compact set $C$ we may assume that $t \in\left[M_{1}, M_{2}\right]$ for some $M_{1}, M_{2} \in \mathbb{R}$. We now have that $\left(\psi_{\ell}\left\lceil B_{L}\right)^{-1}(C)=(C+\ell) \cap B_{L}\right.$ is a closed subset of the compactum $C+\left[M_{1}, M_{2}\right] u$ and hence the set is compact. The proof is complete.

Lemma 7. Let $k \in \mathbb{N}$ with $k<\operatorname{dim} \mathbb{V}$ and let $B$ be closed and convex in $\mathbb{V}$. If $P \in \mathcal{G}_{k}(\mathbb{V})$ is such that $\psi_{P}\left\lceil B\right.$ is proper, then $\operatorname{codim} \mathcal{L}_{B}=\operatorname{codim} \mathcal{L}_{\psi_{P}(B)}$.

Proof. Let $\mathbf{0} \in B$ and $B_{P}=\psi_{P}(B)$. Observe that $P \cap \mathcal{L}_{B}=\{\mathbf{0}\}$ because $\psi_{P}\lceil B$ is proper. We consider two cases.

Case I. Let $k=1$. Consider $V=P+\mathcal{L}_{B}$. Then $\mathcal{L}_{B}$ is a hyperplane in $V$ because $P \cap \mathcal{L}_{B}=\{\mathbf{0}\}$. Observe that $\psi_{P}\left(\mathcal{L}_{B}\right)=\psi_{P}(V)$ is then also a hyperplane in $V$ and hence

$$
\operatorname{codim} \mathcal{L}_{B}=1+\operatorname{codim} V=\operatorname{codim} \psi_{P}\left(\mathcal{L}_{B}\right) .
$$

It now suffices to prove the following claim.

Claim 1. $\mathcal{L}_{B_{P}}=\psi_{P}\left(\mathcal{L}_{B}\right)$.

Proof of Claim. Clearly, we have that $\psi_{P}\left(\mathcal{L}_{B}\right) \subset \mathcal{L}_{B_{P}}$. Thus we need to show that $\mathcal{L}_{B_{P}} \subset \psi_{P}\left(\mathcal{L}_{B}\right)$. Striving for a contradiction, we assume that there is a 1subspace $q$ in $\mathcal{L}_{B_{P}}$ such that $q \perp \psi_{P}\left(\mathcal{L}_{B}\right)=\psi_{P}(V)$. Observe that $q \perp P$ because $q \subset B_{P} \subset P^{\perp}$. Thus $q \perp V$. Set $M=q+P$ and $B^{\prime}=M \cap B$ and note that $M \cap \mathcal{L}_{B}=\{\mathbf{0}\}$ and $\psi_{P}\left(B^{\prime}\right)=q$. Choose an $(x, y)$-coordinate system for the 2-plane $M$ such that $q$ is the $x$-axis and $P$ is the $y$-axis. Since $\psi_{P}\left\lceil B^{\prime}\right.$ is a proper map onto $q$ there are functions $f^{-}, f^{+}: \mathbb{R} \rightarrow \mathbb{R}$ such that $f^{-} \leq f^{+}$and $B^{\prime}=\left\{(x, y) \in M: f^{-}(x) \leq y \leq f^{+}(x)\right\}$. We may assume that $f^{-}(0)=0$. By the Hahn-Banach Theorem there are for each $a \in \mathbb{R}$ linear functions $g_{a}^{-}, g_{a}^{+}: \mathbb{R} \rightarrow \mathbb{R}$ such that $g_{a}^{-}(a)=f^{-}(a), g_{a}^{+}(a)=f^{+}(a)$, and $g_{a}^{-} \leq f^{-} \leq f^{+} \leq g_{a}^{+}$. Consequently, $g_{a}^{-} \leq g_{b}^{+}$for all $a, b \in \mathbb{R}$ and hence all the functions $g_{a}^{-}$and $g_{b}^{+}$have the same slope $m$. Thus $g_{a}^{-}(x)=m(x-a)+f^{-}(a)$ for $a, x \in \mathbb{R}$. If $a \in \mathbb{R}$, then $g_{a}^{-}(0)=$ $-m a+f^{-}(a) \leq f^{-}(0)=0$ and $g_{0}^{-}(a)=m a \leq f^{-}(a)$; thus $f^{-}(a)=m a$. This means that the graph of $f^{-}$is a line that is contained in $B$, which violates the fact that $M \cap \mathcal{L}_{B}=\{\mathbf{0}\}$.

Case II. Now, by an easy induction, we deal with the general case. Let $\left\{e_{1}, e_{2}, \ldots\right.$, $\left.e_{k}\right\}$ be an orthogonal basis for $P$. Set $\ell_{i}=\mathbb{R} e_{i}$ for $1 \leq i \leq k$. Inductively, we define $B_{0}=B$ and $B_{i}=\psi_{\ell_{i}}\left(B_{i-1}\right)$ for $1 \leq i \leq k$. We have that $\psi_{P}=\psi_{\ell_{k}} \circ \ldots \psi_{\ell_{2}} \circ \psi_{\ell_{1}}$ and by Lemma 4 we have that every $\psi_{\ell_{i}} \uparrow B_{i-1}$ is proper. By Case I and induction we get that $\operatorname{codim} \mathcal{L}_{B}=\operatorname{codim} \mathcal{L}_{B_{1}}=\cdots=\operatorname{codim} \mathcal{L}_{B_{k}}$. Thus $\operatorname{codim} \mathcal{L}_{\psi_{P}(B)}=\operatorname{codim} \mathcal{L}_{B}$ and the proof is complete.

The following lemma is an improvement over [3, Lemma 8] with a similar proof.

Lemma 8. Let $k \in \mathbb{N}$ with $k<\operatorname{dim} \mathbb{V}$, let $B$ be a closed and convex set in $\mathbb{V}$, and let $\mathcal{P}$ be an open subset of $\mathcal{G}_{k}(\mathbb{V})$. Suppose that $x \in \mathbb{V}$ is such that there are two distinct supporting hyperplanes $H_{1}$ and $H_{2}$ at $x$ to $B$, at least one of which is consistent with $\mathcal{P}$. Then there are a supporting hyperplane $H$ to $B$ at $x$, a $P \in \mathcal{P}$, and a line $\ell$ through 0 such that $\ell \subset P, x+P \subset H, \psi_{\ell}\lceil B: B \rightarrow \mathbb{V}$ is proper, and $H \cap B \subset H_{1} \cap H_{2} \subset H$. 
Proof. Choose a coordinate system for $\mathbb{V}$ such that $x=\mathbf{0}$. By assumption there are distinct supporting hyperplanes $H_{1}, H_{2}$ to $B$ at $\mathbf{0}$, and there are $v_{1}, v_{2} \in \mathbb{S}$ such that $v_{1} \neq \pm v_{2}, v_{1} \perp H_{1}, v_{2} \perp H_{2}$ and $v_{i} \cdot y \leq 0$ for every $y \in B$ and $i=1,2$. Moreover, we may assume that there is a $P_{1} \in \mathcal{P}$ with $P_{1} \subset H_{1}$. Since $H_{1} \neq H_{2}$ we have that $L=H_{1} \cap H_{2}$ has codimension 2. Since $\operatorname{dim}\left(P_{1} \cap L\right)=\operatorname{dim}\left(P_{1} \cap H_{2}\right) \geq k-1$ we can select a subspace $P^{\prime}$ of $P_{1} \cap L$ with $\operatorname{dim} P^{\prime}=k-1$. Select a basis $\left\{e_{1}, \ldots, e_{k}\right\}$ for $P_{1}$ such that $\left\{e_{1}, \ldots, e_{k-1}\right\}$ is a basis for $P^{\prime}$. With Lemma 2 we can find an $\varepsilon>0$ such that $\left[\left\{e_{1}, \ldots, e_{k-1}, u\right\}\right] \in \mathcal{P}$ for each $u$ with $\left\|e_{k}-u\right\|<\varepsilon$. We can select a vector $e_{k}^{\prime} \in H_{1} \backslash L$ such that $\left\|e_{k}^{\prime}-e_{k}\right\|<\varepsilon$. Then obviously $e_{k}^{\prime} \cdot v_{2} \neq 0$ and we may assume that $e_{k}^{\prime} \cdot v_{2}>0$ because we may replace $e_{k}$ and $e_{k}^{\prime}$ by their opposite vectors. Note that $e_{k}^{\prime} \cdot v_{1}=0$. Using Lemma 2 in the same way as above, we can select an approximation $e$ to $e_{k}^{\prime}$ such that $P=\left[\left\{e_{1}, \ldots, e_{k-1}, e\right\}\right] \in \mathcal{P}, e \cdot v_{2}>0$, and $e \cdot v_{1}<0$. Let $\ell$ be the line $\mathbb{R} e$ in $P$. Let

$$
a=\frac{v_{2}}{e \cdot v_{2}}-\frac{v_{1}}{e \cdot v_{1}}
$$

and note that $a \neq \mathbf{0}$ because $v_{1} \neq \pm v_{2}$ and that $a \cdot e=0$. Define $H=\{y \in \mathbb{V}$ : $y \cdot a=0\}$ and note that $H=L+\ell$ and therefore, $P \subset H$. If $y \in B$, then $y \cdot v_{1} \leq 0$ and $y \cdot v_{2} \leq 0$; so also $y \cdot a \leq 0$, and hence $H$ is a supporting hyperplane to $B$.

Let $z \in \mathbb{V}$ and let $y=z+\alpha e$ be in $B$ for some $\alpha \in \mathbb{R}$. Since $v_{j} \cdot y \leq 0$ for $j=1,2$ we get that $\alpha e \cdot v_{j} \leq-z \cdot v_{j} \leq\|z\|$. Since $e \cdot v_{1}<0$ and $e \cdot v_{2}>0$ we have

$$
\frac{-z \cdot v_{1}}{e \cdot v_{1}} \leq \alpha \leq \frac{-z \cdot v_{2}}{e \cdot v_{2}} .
$$

Thus we see that every fibre of $\psi_{\ell} \uparrow B$ is bounded and therefore the map is proper by Lemma 6. If we assume in addition that $y \in H$, then we may take $z \in L$. Consequently, $\alpha=0$ since $z \cdot v_{i}=0$ for $i=1,2$. Hence $H \cap B \subset L=H_{1} \cap H_{2}$. The proof is complete.

Lemma 9. Let $k \in \mathbb{N}$ with $k<\operatorname{dim} \mathbb{V}$ and let $C$ be closed in $\mathbb{V}$. If $P \in \mathcal{G}_{k}(\mathbb{V})$ and $w \in \mathbb{V}$ are such that $\psi_{P}\lceil\overline{\langle C\rangle}$ is proper and $(w+P) \cap C=\emptyset$, then there is a neighbourhood $\mathcal{U}$ of $P$ such that $\left(w+P^{\prime}\right) \cap C=\emptyset$ for each $P^{\prime} \in \mathcal{U}$.

Proof. We may put $w=\mathbf{0}$. Striving for a contradiction we assume that the conclusion is false, which means that we can find a sequence $P_{1}, P_{2}, \ldots \in \mathcal{G}_{k}(\mathbb{V})$ such that $\lim _{i \rightarrow \infty} P_{i}=P$ and $P_{i} \cap C \neq \emptyset$ for each $i \in \mathbb{N}$. Select $x_{i} \in P_{i} \cap C$ for $i \in \mathbb{N}$.

Case I: $\left\{x_{i}: i \in \mathbb{N}\right\}$ is bounded. Let $M$ be such that $\mathbb{B}_{M}=\{x \in \mathbb{V}:\|x\| \leq M\}$ contains every $x_{i}$. By the definition of $\rho$ we may select for every $i \in \mathbb{N}$ a point $y_{i}$ in $P \cap \mathbb{B}_{M}$ such that $d\left(x_{i}, y_{i}\right) \leq M \rho\left(P_{i}, P\right)$ and hence $\lim _{i \rightarrow \infty} d\left(x_{i}, y_{i}\right)=0$. Since $P \cap \mathbb{B}_{M}$ is compact we may assume (by passing to a subsequence) that $\lim _{i \rightarrow \infty} y_{i}=$ $y \in P \cap \mathbb{B}_{M}$. Consequently, we also have $\lim _{i \rightarrow \infty} x_{i}=y$ and hence $y \in P \cap C$ because $C$ is closed. Thus we have a contradiction with the assumption $(w+P) \cap C=\emptyset$.

Case II: $\left\{x_{i}: i \in \mathbb{N}\right\}$ is unbounded. Then we may assume that $\lim _{i \rightarrow \infty}\left\|x_{i}\right\|=\infty$ and $x_{i} \neq \mathbf{0}$ for each $i$. By the same argument as employed in Case I, we may assume that there is a (unit vector) $u \in P$ such that $\lim _{i \rightarrow \infty} x_{i} /\left\|x_{i}\right\|=u$. Let $z \in C$ and $t \geq 0$ and consider $z+t u \in z+P$. Defining $v_{i}=z+\frac{t}{\left\|x_{i}\right\|}\left(x_{i}-z\right)$ we find $\lim _{i \rightarrow \infty} v_{i}=z+t u$. If $\left\|x_{i}\right\| \geq t$, then $v_{i} \in \overline{\langle C\rangle}$; thus $z+t u \in \overline{\langle C\rangle}$. We now have that $\psi_{P}\lceil\overline{\langle C\rangle}$ has an unbounded fibre which violates properness.

Lemma 10. Let $k \in \mathbb{N}$ with $k<\operatorname{dim} \mathbb{V}$ and let $B$ be a closed and convex set in $\mathbb{V}$. Then $\left\{P \in \mathcal{G}_{k}(\mathbb{V}): \psi_{P}\lceil B\right.$ is proper $\}$ is open in $\mathcal{G}_{k}(\mathbb{V})$. 
Proof. Let $P \in \mathcal{G}_{k}(\mathbb{V})$ such that $\psi_{P}\lceil B$ is proper. We may assume that $\mathbf{0} \in B$. Since $\psi_{P}\lceil B$ is proper, $P \cap B$ is bounded, so there is an $M>0$ such that $P$ is disjoint from $C=\{x \in B:\|x\| \geq M\}$. Since $\overline{\langle C\rangle}$ is a closed subset of $B$ we have that also $\left.\psi_{P}\right\rceil \overline{\langle C\rangle}$ is proper. Thus by Lemma 9 we have that there is a neighbourhood $\mathcal{U}$ of $P$ such that $P^{\prime} \cap C=\emptyset$ for each $P^{\prime} \in \mathcal{U}$. If $P^{\prime} \in \mathcal{U}$, then $P^{\prime} \cap B$ is bounded because $B \backslash C$ is bounded. Thus by Lemma $\left[6\right.$ we have that $\psi_{P^{\prime}}\lceil B$ is proper.

Lemma 11. Let $k \in \mathbb{N}$ with $k<\operatorname{dim} \mathbb{V}$, let $\mathcal{P}$ be an open subset of $\mathcal{G}_{k}(\mathbb{V})$, and let $B$ be a closed and convex set in $\mathbb{V}$ that contains no $k$-hyperplane. If $P \in \mathcal{P}$ and $w \in \mathbb{V}$ are such that $(w+P) \cap B=\emptyset$, then there is a nonempty open subset $\mathcal{U}$ of $\mathcal{P}$ such that $\psi_{L} \backslash B$ is proper and $(w+L) \cap B=\emptyset$ for every $L \in \mathcal{U}$.

Proof. Let $P \in \mathcal{P}$ and $w \in \mathbb{V}$ be such that $(w+P) \cap B=\emptyset$. We may assume that $w=\mathbf{0}$. According to Lemmas 9 and 10 it suffices to prove that there is a $P^{\prime} \in \mathcal{P}$ such that $\psi_{P^{\prime}}\left\lceil B\right.$ is proper and $P^{\prime} \cap B=\emptyset$. If $B^{\circ}=\emptyset$ (which can happen only if $B$ is infinite-dimensional), then the existence of $P^{\prime}$ is guaranteed by [4, Lemma 20]. So we may assume that $B^{\circ} \neq \emptyset$.

Define

$$
\begin{gathered}
\mathcal{A}=\left\{L: L \text { is a linear subspace of some } P^{\prime} \in \mathcal{P}\right. \text { such that } \\
\psi_{L}\left\lceil B \text { is proper and } P^{\prime} \cap B=\emptyset\right\} .
\end{gathered}
$$

Note that $\{\mathbf{0}\} \in \mathcal{A}$ because $\psi_{\{\mathbf{0}\}}\lceil B$ is the identity map and $\{\mathbf{0}\} \subset P$. We may define $l=\max \{\operatorname{dim} L: L \in \mathcal{A}\}$. It suffices to show that $l=k$, so let us assume that $l<k$. Choose linear subspaces $P_{1} \in \mathcal{P}$ and $L \subset P_{1}$ such that $\operatorname{dim} L=l, \psi_{L}\lceil B$ is proper, and $P_{1} \cap B=\emptyset$. Define

$$
B_{L}=\psi_{L}(B) \quad \text { and } \quad \mathcal{P}_{L}=\left\{N \in \mathcal{G}_{k-l}\left(L^{\perp}\right): N+L \in \mathcal{P}\right\} .
$$

Clearly, $N=\psi_{L}\left(P_{1}\right) \in \mathcal{P}_{L}, \mathbf{0}=\psi_{L}(\mathbf{0})$ and $B_{L}$ is closed. By [3, Corollary 4] and 4. Remark 1], $\mathcal{P}_{L}$ is open in $\mathcal{G}_{k-l}\left(L^{\perp}\right)$. Notice that $\left(B_{L}\right)^{\circ} \neq \emptyset$ and $\psi_{N}\left(B_{L}\right)^{\circ} \neq \emptyset$ because $B^{\circ} \neq \emptyset$ and projections are open maps. Since $\psi_{P_{1}}(B)^{\circ} \neq \emptyset$ and $\mathbf{0}=$ $\psi_{P_{1}}(\mathbf{0}) \notin \psi_{P_{1}}(B)$ there is a supporting hyperplane $H$ in $\left(P_{1}\right)^{\perp}$ at $\mathbf{0}$ to $\psi_{N}\left(B_{L}\right)$. Set $H_{1}=H+N$ and observe that $H_{1}$ is a supporting hyperplane in $L^{\perp}$ at $\mathbf{0}$ to $B_{L}$.

We will find a supporting hyperplane $H_{2}$ at $\mathbf{0}$ to $B_{L}$ in $L^{\perp}$ such that $H_{1} \neq H_{2}$. If $d\left(H_{1}, B_{L}\right)=0$, then there exists a supporting hyperplane $H_{2}$ at $\mathbf{0}$ to $B_{L}$ such that $d\left(H_{2}, B_{L}\right)=d\left(\mathbf{0}, B_{L}\right)>0$; see [12, p.347]. Now let $d\left(H_{1}, B_{L}\right)>0$. By Lemma 7 we have that $k<\operatorname{codim} \mathcal{L}_{B}=\operatorname{codim} \mathcal{L}_{B_{L}}$. Thus also $B_{L}$ does not contain a $k$-hyperplane of $\mathbb{V}$. On the other hand, the $(\ell+1)$-hyperplane $H_{1}$ contains a $k$ hyperplane since $\ell+1 \leq k$. Therefore, we can find a $u \in \mathbb{S} \cap H_{1}$ such that $z+\mathbb{R}^{+} u \not \subset B_{L}$ for $z \in B_{L}$, where $R^{+}=[0, \infty)$. Note that $\left(z+\mathbb{R}^{+} u\right) \cap B_{L}$ is bounded because $z \in B_{L}$ and $B_{L}$ is convex. Let $C$ be the closed convex set $\left\{x \in B_{L}: x \cdot u \geq 0\right\}$. If $z+t u \in C$, then $(z+t u) \cdot u \geq 0$; hence $t \geq-z \cdot u$. This means that $(z+\mathbb{R} u) \cap C$ is bounded and hence $\psi_{\mathbb{R} u} \uparrow C$ is proper by Lemma 6 , Note that $\mathbb{R} u \cap B_{L}=\emptyset$ because $d\left(H_{1}, B_{L}\right)>0$. Thus by Lemma 9 there is a neighbourhood $\mathcal{U}$ of $\mathbb{R} u$ in $\mathcal{G}_{1}\left(L^{\perp}\right)$ such that every $\ell \in \mathcal{U}$ is disjoint from $C$. Let $v$ be a vector in $L^{\perp}$ that is perpendicular to $H_{1}$ and on the same side of $H_{1}$ as $B_{L}$ so $y \cdot v>0$ for each $y \in B_{L}$. We may assume that $v$ is small enough so that the line $\mathbb{R}(u+v)$ is in $\mathcal{U}$. We verify that $\mathbb{R}(u+v) \cap B_{L}=\emptyset$. Let $x=t(u+v)$ and hence $x \notin C$. If $t \geq 0$, then $x \cdot u=t \geq 0$; thus $x \notin B_{L}$. If $t<0$, then $x \cdot v=t\|v\|^{2}<0$; thus $x \notin B_{L}$. Since $\left(B_{L}\right)^{\circ} \neq \emptyset$ we can find with the Hahn-Banach Theorem a 
hyperplane $H_{2}$ in $L^{\perp}$ containing $\mathbb{R}(u+v)$ such that $H_{2}$ supports $B_{L}$. Observe that $u+v \in H_{2} \backslash H_{1}$, so $H_{1} \neq H_{2}$.

We have shown that there are at least two supporting hyperplanes at $\mathbf{0}$ to $B_{L}$ in $L^{\perp}$, one of which is consistent with $\mathcal{P}_{L}$. In $L^{\perp}$ we can now apply Lemma 8 to $B_{L}, \mathcal{P}_{L}$, and $\mathbf{0}$ to get a supporting hyperplane $H$ to $B_{L}$ at $\mathbf{0}$, an $N^{\prime} \in \mathcal{P}_{L}$, and a line $\ell \subset N^{\prime}$ such that $\mathbf{0} \in \ell, N^{\prime} \subset H, \psi_{\ell}\left\lceil B_{L}\right.$ is proper and $H \cap B_{L} \subset H_{1} \cap H_{2}$. Furthermore, since $d\left(H_{i}, B_{L}\right)>0$ for some $i \in\{1,2\}$ we get that $H \cap B_{L}=\emptyset$. Set $L^{\prime}=L+\ell$ and $P_{1}^{\prime}=L+N^{\prime}$. Now, we have that $P_{1}^{\prime} \cap B \subset(L+H) \cap B=\emptyset$. In addition, we have that $\psi_{L}\left\lceil B\right.$ and $\psi_{\ell}\left\lceil B_{L}\right.$ are both proper. Therefore, $\psi_{L^{\prime}} \uparrow B$ is proper by Lemma 4. We now have that $L^{\prime} \in \mathcal{A}$ and $\operatorname{dim} L^{\prime}=l+1$, which contradicts the maximality of $l$. That completes the proof.

Lemma 12. Let $k \in \mathbb{N}$ with $k<\operatorname{dim} \mathbb{V}$, let $B$ and $C$ be convex closed subsets of $\mathbb{V}$, and let $\mathcal{P}$ be a subset of $\mathcal{G}_{k}(\mathbb{V})$ such that $\mathcal{P} \subset \operatorname{int} \overline{\mathcal{P}}$ and $C$ is a weak $\mathcal{P}$-imitation of $B$. Then $\mathcal{E}^{k}(C, \mathcal{P})=\mathcal{E}^{k}(B, \mathcal{P})$. If, in addition, $B$ does not contain a $k$-hyperplane and $B$ is not an (int $\overline{\mathcal{P}}$ )-imitation of $\mathbb{V}$, then also $C$ does not contain a $k$-hyperplane and $B$ and $C$ have identical (derived) $\mathcal{P}$-faces.

Proof. Note that $B$ and $C$ have identical supporting hyperplanes that are consistent with $\mathcal{P}$ by Remark 1

Claim 2. If $B$ does not contain a $k$-hyperplane, then every $\mathcal{P}$-face of $C$ is contained in $B$.

Proof of Claim. Let $H_{1}$ be a supporting hyperplane to $C$ that is consistent with $\mathcal{P}$. Striving for a contradiction we assume that $H_{1} \cap C \backslash B \neq \emptyset$. Choose a coordinate system such that $\mathbf{0} \in H_{1} \cap C \backslash B$. Let $H_{2}$ be the (unique) hyperplane through $\mathbf{0}$ with $d\left(H_{2}, B\right)=d(\mathbf{0}, B)>0$; see [12, p. 347]. Let $P_{1} \in \mathcal{P}$ be such that $P_{1} \subset H_{1}$. Since $\psi_{P_{1}}(\mathbf{0}) \in \psi_{P_{1}}(C) \subset \overline{\psi_{P_{1}}(B)}$ we have that $d\left(P_{1}, B\right)=0$ and hence $d\left(H_{1}, B\right)=0$. Thus we have that $H_{1} \neq H_{2}$ and we can find a supporting hyperplane $H$ at $\mathbf{0}$ and a $P \in$ int $\overline{\mathcal{P}}$ with $P \subset H$ and $H \cap B \subset H_{1} \cap H_{2}$ by Lemma 8. We have that $P \cap B=\emptyset$ because $H_{2} \cap B=\emptyset$. Now, by Lemma 11, we find a nonempty open subset $\mathcal{U}$ of int $\overline{\mathcal{P}}$ such that for every $L \in \mathcal{U}$ we have that $\psi_{L}\lceil B$ is proper and $\psi_{L}(\mathbf{0}) \notin \psi_{L}(B)=\overline{\psi_{L}(B)}$. Let $L \in \mathcal{P} \cap \mathcal{U}$. Then $\psi_{L}(\mathbf{0}) \in \psi_{L}(C) \backslash \overline{\psi_{L}(B)}$, which contradicts the premise that $C$ is a weak $\mathcal{P}$-imitation of $B$.

Claim 3. If $B$ does not contain a $k$-hyperplane and $B$ is not an (int $\overline{\mathcal{P}}$ )-imitation of $\mathbb{V}$, then also $C$ does not contain a $k$-hyperplane.

Proof of Claim. By Lemma 11 there is a $P \in \mathcal{P}$ such that $\psi_{P}\lceil B$ is proper and $\psi_{P}(B) \neq P^{\perp}$. Select a $w \in P^{\perp} \backslash \psi_{P}(B)$ and note that $\psi_{P}(B)$ is closed and convex; thus there exists by [12, p. 347] an $x \in \psi_{P}(B)$ with $d(w, x)=d\left(w, \psi_{P}(B)\right)$. Since we are in a Hilbert space the hyperplane $H$ in $P^{\perp}$ through $x$ that is perpendicular to the vector $w-x$ supports $\psi_{P}(B)$. Note that then $H+P$ is a hyperplane in $\mathbb{V}$ such that $(H+P) \cap B$ is a $\mathcal{P}$-face of $B$. Put $D=\overline{\langle C \cup B\rangle}$ and note that $D$ is a weak $\mathcal{P}$-imitation of $B$ by Lemma 3 . Then $H+P$ supports $D$ as well and $(H+P) \cap D$ is a $\mathcal{P}$-face of $D$ because it contains $(H+P) \cap B$ and is thus nonempty. By Claim 2 we now have that $(H+P) \cap D \subset B$. If $C$ contains a $k$-hyperplane, then so does $D$ and hence every face of $D$ contains a $k$-hyperplane by Lemma 5 . Consequently, $B$ contains a $k$-hyperplane, contradicting an assumption. We may conclude that $C$ does not contain a $k$-hyperplane of $\mathbb{V}$. 
To prove that $\mathcal{E}^{k}(C, \mathcal{P})=\mathcal{E}^{k}(B, \mathcal{P})$ we consider two cases.

Case I: Neither $B$ nor $C$ contains a $k$-hyperplane. Then by Claim 2 and the fact that $B$ and $C$ have the same supporting hyperplanes we have that $B$ and $C$ have the same $\mathcal{P}$-faces (and hence the same derived $\mathcal{P}$-faces). Therefore $\mathcal{E}^{k}(C, \mathcal{P})=$ $\mathcal{E}^{k}(B, \mathcal{P})$.

Case II: Either $B$ or $C$ contains a $k$-hyperplane. By symmetry we may assume that $C$ contains a $k$-hyperplane. Then by Lemma 5 every derived face $F$ of $C$ contains a $k$-hyperplane and hence $\operatorname{codim} F \leq k$. Since derived $\mathcal{P}$-faces are derived faces we have $\mathcal{E}^{k}(C, \mathcal{P})=\emptyset$. If $B$ contains a $k$-hyperplane, then also $\mathcal{E}^{k}(B, \mathcal{P})=\emptyset$ and we are done. Assume now that $B$ does not contain a $k$-hyperplane. Then by Claim 3 and $\mathcal{P} \subset \operatorname{int} \overline{\mathcal{P}}$ we have that $B$ is a $\mathcal{P}$-imitation of $\mathbb{V}$ and hence $B$ has no $\mathcal{P}$-faces by Remark 1. Consequently, $\mathcal{E}^{k}(B, \mathcal{P})=\emptyset$ and Case II is finished.

Finally, for the last part of the lemma, assume that $B$ does not contain a $k$ hyperplane and $B$ is not a (int $\overline{\mathcal{P}}$ )-imitation of $\mathbb{V}$. Then by Claim 3 also $C$ does not contain a $k$-hyperplane and we are in Case I. Thus $B$ and $C$ have the same (derived) $\mathcal{P}$-faces.

In the situation of Lemma 12, if $\mathcal{P}$ is open, then, by the proof of [3, Lemma 9], we have that $B$ and $C$ have the same $\mathcal{P}$-faces. If we merely know that $\mathcal{P} \subset$ int $\overline{\mathcal{P}}$, then $B$ and $C$ may not have the same $\mathcal{P}$-faces as the following example shows. So it is essential to consider $k$-hyperplanes in Lemma 12 .

Example 1. Let $\mathbb{V}$ be $\mathbb{R}^{3}$ with the usual $x y z$ coordinate system. Let $B$ be the $x$-axis, $C$ the $y$-axis, and $\mathcal{P}=\left\{P \in \mathcal{G}_{2}\left(\mathbb{R}^{3}\right): B \not \subset P, C \not \subset P\right\} \cup\{x y$-plane $\}$. Then, clearly, $\overline{\mathcal{P}}=\mathcal{G}_{2}\left(\mathbb{R}^{3}\right)$. In addition, $\psi_{P}(C)=\psi_{P}(B)=\{\mathbf{0}\}$ if $P=x y$-plane and $\psi_{P}(C)=\psi_{P}(B)=P^{\perp}$ if $P \in \mathcal{P} \backslash\{x y$-plane $\}$. Thus $B$ and $C$ are $\mathcal{P}$-imitations of each other. However, $B$ and $C$ do not have the same $\mathcal{P}$-faces since if $P$ stands for the $x y$-plane, then $B=P \cap B \neq P \cap C=C$.

\section{The main theorems}

Theorem 1 follows immediately from the following theorem and [3, Theorem 18].

Theorem 13. Let $k \in \mathbb{N}$ with $k<\operatorname{dim} \mathbb{V}$, let $B$ be a closed convex subset of $\mathbb{V}$ that contains no $k$-hyperplane, and let $\mathcal{P}$ be a subset of $\mathcal{G}_{k}(\mathbb{V})$ such that $(w+P) \cap B=\emptyset$ for a $P \in \operatorname{int} \overline{\mathcal{P}}$ and $a w \in \mathbb{V}$. If $C$ is a closed weak $\mathcal{P}$-imitation of $B$, then there is a nonempty open subset $\mathcal{U}$ of int $\overline{\mathcal{P}}$ such that $C$ is a $\mathcal{U}$-imitation of $B$ and for every $\left.L \in \mathcal{U}, \psi_{L}\right\rceil \overline{\langle B \cup C\rangle}$ is proper and $(w+L) \cap \overline{\langle B \cup C\rangle}=\emptyset$.

Proof. Let $\hat{\mathcal{P}}=$ int $\overline{\mathcal{P}}$. By Lemma 11 there is a nonempty open subset $\mathcal{V}$ of $\hat{\mathcal{P}}$ such that for every $L \in \mathcal{V}$ we have that $\psi_{L}\lceil B$ is proper and $(w+L) \cap B=$ $\emptyset$. Consider $D=\overline{\langle B \cup C\rangle}$ and note that $B, C$, and $D$ are weak $\mathcal{P}$-imitations of each other by Lemma 3. Note that by Lemma $12 D$ also cannot contain a $k$ hyperplane. Furthermore, for every $L \in \mathcal{V} \cap \mathcal{P}$ we have that $\psi_{L}(B)=\overline{\psi_{L}(C)}=$ $\overline{\psi_{L}(D)}$. Therefore, we get $(w+L) \cap D=\emptyset$ for $L \in \mathcal{V} \cap \mathcal{P}$. By Lemma 11, there is an open subset $\mathcal{U}$ of $\mathcal{V}$ such that for every $L^{\prime} \in \mathcal{U}$ we have that $\psi_{L^{\prime}}\lceil D$ is proper and $\left(w+L^{\prime}\right) \cap D=\emptyset$. Now let us show that $C$ is a $\mathcal{U}$-imitation of $B$. Pick an $L \in \mathcal{U}$ and assume that there is a $w^{\prime} \in B$ such that $\psi_{L}\left(w^{\prime}\right) \notin \psi_{L}(C)$. Since $\overline{\langle C\rangle} \subset D$ we have that $\psi_{L} \uparrow \overline{\langle C\rangle}$ is proper so that we may apply Lemma 9 to get a nonempty open subset $\mathcal{U}^{\prime}$ of $\mathcal{U}$ such that $\left(w^{\prime}+L^{\prime}\right) \cap C=\emptyset$ for every $L^{\prime} \in \mathcal{U}^{\prime}$. Select an $L^{\prime} \in \mathcal{P} \cap \mathcal{U}^{\prime}$ and note that $\psi_{L^{\prime}}\left(w^{\prime}\right) \notin \psi_{L^{\prime}}(C)=\overline{\psi_{L^{\prime}}(C)}$, which contradicts the fact 
that $C$ is a weak $\mathcal{P}$-imitation of $B$. Therefore $\psi_{L}(B) \subset \psi_{L}(C)$ for every $L \in \mathcal{U}$. By a symmetric argument we have that $\psi_{L}(C) \subset \psi_{L}(B)$ for each $L \in \mathcal{U}$. Hence $C$ is a $\mathcal{U}$-imitation of $B$.

We have the following improvement over [3, Theorem 1].

Theorem 14. Let $k, n \in \mathbb{N}$ with $k<n$, let $C$ be a closed nonconvex subset of $\mathbb{R}^{n}$, and let $\mathcal{P} \subset \mathcal{G}_{n-k}\left(\mathbb{R}^{n}\right)$. Let $\psi_{P^{*}}(\langle C\rangle) \neq\left(P^{*}\right)^{\perp}$ for some $P^{*} \in \operatorname{int} \overline{\mathcal{P}}$ and let $\overline{\psi_{P}(C)}$ be convex for every $P \in \mathcal{P}$. If $\langle C\rangle$ contains no $k$-plane, then $C$ contains a closed set that is homeomorphic to either

(i) $\mathbb{R}^{k-1}$ or

(ii) $S^{i} \times \mathbb{R}^{k-i-1}$ for some $i \in\{1,2, \ldots, k-1\}$.

Proof. Set $B=\overline{\langle C\rangle}$ and apply Theorem[1 in analogy to the proof of [3, Theorem 1].

The following result improves upon both [3, Theorem 16] and [2, Theorem 9].

Theorem 15. Let $k \in \mathbb{N}$ with $k<\operatorname{dim} \mathbb{V}$, let $B$ be a closed and convex set in $\mathbb{V}$, and let $\mathcal{P}$ be a subset of $\mathcal{G}_{k}(\mathbb{V})$ such that $\mathcal{P} \subset \operatorname{int} \overline{\mathcal{P}}$. If $C$ is a closed set that is a weak $\mathcal{P}$-imitation of $B$, then $\mathcal{E}^{k}(B, \mathcal{P}) \subset C$.

Proof. Let $C$ be a closed set in $\mathbb{V}$ such that for every $P \in \mathcal{P}, \overline{\psi_{P}(C)}=\overline{\psi_{P}(B)}$. Consider the closed convex set $D=\overline{\langle C\rangle}$ and note that $B, C$, and $D$ are all weak $\mathcal{P}$ imitations of each other by Lemma 3, According to Lemma 12 we have $\mathcal{E}^{k}(B, \mathcal{P})=$ $\mathcal{E}^{k}(D, \mathcal{P})$. To prove that $\mathcal{E}^{k}(D, \mathcal{P}) \subset C$ it suffices to show that every derived $\mathcal{P}$-face of $D$ with codimension greater than $k$ is contained in the closed set $C$.

Let $F$ be such a derived $\mathcal{P}$-face of $D$. Striving for a contradiction we suppose that $F \backslash C \neq \emptyset$. We may choose a coordinate system such that $\mathbf{0} \in F \backslash C$. Precisely as in the proof of [3, Theorem 16] we can find an $N \in \operatorname{int} \overline{\mathcal{P}}$ such that $N \cap C=\emptyset$ and $N \cap D$ is bounded. Thus we have that $\psi_{N}\lceil D$ is proper by Lemma 6 . According to Lemmas 9 and 10 there is a neighbourhood $\mathcal{V}$ of $N$ in int $\overline{\mathcal{P}}$ such that $\psi_{L}\lceil D$ is proper and $L \cap C=\emptyset$ for every $L \in \mathcal{V}$. Select an $L \in \mathcal{U} \cap \mathcal{P}$ and note that $\psi_{L}(C)=\overline{\psi_{L}(C)}$ because $C$ is a closed subset of $D$. We now have that $\mathbf{0} \in \psi_{L}(D) \backslash \overline{\psi_{L}(C)}$, which violates the fact that $C$ is a weak $\mathcal{P}$-imitation of $D$.

We finish by displaying that the points in $\mathcal{E}^{k}(B, \mathcal{P})$ (the $\mathcal{P}$-extremal points of $B$ ) are precisely the points that are included in every closed $\mathcal{P}$-imitation of $B$ whenever $\mathcal{P} \subset \operatorname{int} \overline{\mathcal{P}} \subset \mathcal{G}_{k}\left(\mathbb{R}^{n}\right)$. This is an improvement over [3, Theorem 23].

Theorem 16. Let $k, n \in \mathbb{N}$ with $k<n$, let $B$ be a closed convex set in $\mathbb{R}^{n}$ with $\operatorname{codim} B \neq k$, and let $\mathcal{P} \subset \mathcal{G}_{k}\left(\mathbb{R}^{n}\right)$ be such that $\mathcal{P} \subset \operatorname{int} \overline{\mathcal{P}}$. Then

$$
\begin{aligned}
\mathcal{E}^{k}(B, \mathcal{P}) & =\bigcap\{C: C \text { is a closed weak } \mathcal{P} \text {-imitation of } B\} \\
& =\bigcap\{C: C \text { is a closed } \mathcal{P} \text {-imitation of } B\} .
\end{aligned}
$$

Proof. By Theorem 15 we have that

$$
\mathcal{E}^{k}(B, \mathcal{P}) \subset \bigcap\{C: C \text { is a closed weak } \mathcal{P} \text {-imitation of } B\} .
$$

The other parts of the statement are already included in the proof of [3, Theorem 23]. 
Remark 3. It is easily verified that the arguments supporting Remark 7 and Proposition 24 in [3] also work for a $\mathcal{P}$ that satisfies $\mathcal{P} \subset \operatorname{int} \overline{\mathcal{P}}$ instead of openness.

\section{REFERENCES}

[1] S. Barov, J. Cobb and J. J. Dijkstra, On closed sets with convex projections, J. London Math. Soc. 65 (2002), 154-166. MR1875142(2002j:52002)

[2] S. Barov and J. J. Dijkstra, On closed sets with convex projections in Hilbert space, Fund. Math. 197 (2007), 17-33. MR2365881 (2009c:52002)

[3] S. Barov and J. J. Dijkstra, On closed sets with convex projections under narrow sets of directions, Trans. Amer. Math. Soc. 360 (2008), 6525-6543. MR2434297

[4] S. Barov and J. J. Dijkstra, On closed sets in Hilbert space with convex projection under somewhere dense sets of directions, preprint.

[5] C. Bessaga and A. Pełczyński, Selected Topics in Infinite-Dimensional Topology, PWN, Warsaw, 1975. MR0478168 (57:17657)

[6] K. Borsuk, An example of a simple arc in space whose projection in every plane has interior points, Fund. Math. 34 (1947), 272-277. MR0025721(10:54e)

[7] J. Cobb, Raising dimension under all projections, Fund. Math. 144 (1994), 119-128. MR.1273691 (95i:54038)

[8] J. J. Dijkstra, T. L. Goodsell, and D. G. Wright, On compacta with convex projections, Topology Appl. 94 (1999), 67-74. MR.1695348 (2000e:57035)

[9] R. Engelking, General Topology, PWN, Warsaw, 1977. MR0500780 (58:18316b)

[10] R. J. Gardner, Geometric Tomography, Cambridge Univ. Press, Cambridge, 1995. MR.1356221 (96j:52006)

[11] B. Grünbaum, Convex Polytopes, Pure Appl. Math. 16, Interscience, London, 1967. MR0226496 (37:2085)

[12] G. Köthe, Topologische Lineare Räume I, Springer, Berlin, 1960. MR0130551(24:A411)

Institute of Mathematics, Bulgarian Academy of Sciences, 8 Acad. G. Bonchev Street, 1113 Sofia, Bulgaria

E-mail address: stoyu@yahoo.com

Afdeling Wiskunde, Vrije Universiteit, De Boelelaan 1081A, 1081 HV Amsterdam, The Netherlands

E-mail address: dijkstra@cs.vu.nl 